\title{
CYCLIC INEQUALITIES
}

\author{
SHIGERU YAMAGAMI
}

(Communicated by Palle E. T. Jorgensen)

Dedicated to Professor Tomiyama on his sixtieth birthday

\begin{abstract}
We prove a class of cyclic inequalities including the ones that are related with the positivity of Choi's maps in matrix algebras.
\end{abstract}

\section{INTRODUCTION}

Inspired by Nowosad's results on Shapiro's cyclic inequalities, we shall give a proof of another kind of cyclic inequalities that have been conjectured with the relation to the positivity of Choi's maps

$$
\begin{aligned}
& \sum_{j=1}^{n} \frac{x_{j}}{(n-m) x_{j}+x_{j+1}+\cdots+x_{j+m}} \leq 1, \\
& x_{1}>0, \ldots, x_{n}>0,1 \leq m \leq n-1,
\end{aligned}
$$

where the $x_{j}$ 's are cyclically identified as $x_{j+n}=x_{j}$.

The inequality (1), which is proved for $m=1$ in [6], for $m=n-2$ in [1] (the case $m=n-1$ is trivial), and then conjectured in the above form by Nakamura and recently proved for the case $(n, m)=(5,2)$ in [4], now shows that the map $\tau_{m}: M a t_{n}(\mathbb{C}) \rightarrow M_{a t}(\mathbb{C})$ defined by

$$
\tau_{m}(X)=(n-m) \varepsilon(X)+\sum_{j=1}^{m} \varepsilon\left(C^{j} X C^{* j}\right)-X
$$

is positive as pointed out in [6], where $\varepsilon$ denotes the conditional expectation to the diagonal algebra and $C$ the cyclic permutation. See $[4,5]$ for further information.

In this paper, we shall prove more general inequalities: For positive integers $l, m, n$ and a positive real number $s$ such that $s \geq n$ and $1 \leq m \leq n-l$, we have

$$
\sum_{j=1}^{n} \frac{x_{j}}{(s-m) x_{j}+x_{j+l}+\cdots+x_{j+l+m-1}} \leq \frac{n}{s}, \quad x_{1}>0, \ldots, x_{n}>0,
$$

Received by the editors October $9,1991$.

1991 Mathematics Subject Classification. Primary 46L05, 47B55.

Key words and phrases. Cyclic inequality, positive map. 
which is, again by the argument in [6], equivalent to the positivity of the map

$$
X \mapsto(s-m) \varepsilon(X)+\sum_{j=l}^{l+m-1} \varepsilon\left(C^{* j} X C^{j}\right)-\frac{s}{n} X .
$$

\section{PRELIMINARIES}

Let $A$ be a commutative $C^{*}$-algebra containing 1 and let $\varphi$ be a positive linear functional on $A$. Set $A_{h}^{\times}=\left\{x \in A ; x^{*}=x, x\right.$ is invertible in $\left.A\right\}$. For $a \in A_{h}^{\times}$, we denote by $[a]$ the set of elements, say $x$, in $A_{h}^{\times}$such that $x$ and $x^{-1}$ are approximated in the norm topology of $A$ by Laurent polynomials of $a$ (i.e., polynomials of $a$ and $a^{-1}$ ). Note that $[a]$ is a closed subgroup of $A_{h}^{\times}$containing $a$.

The next theorem, due to Nowosad [3, Theorem 1.8], is essential in our proof of (2).

Theorem 1. Let $T: A \rightarrow A$ be a bounded linear operator such that $T\left(x^{*}\right)=$ $T(x)^{*} \forall x \in A$. If the functional $\lambda_{T}: A_{h}^{\times} \ni x \mapsto \varphi\left(x^{-1} T(x)\right)$ has local maximums at $x=1$ and $x=a \in A_{h}^{\times} \backslash \mathbb{R} 1$, then $\lambda_{T}$ is constant on the subset $[a]$.

We shall apply Theorem 1 to the case $A=\overbrace{\mathbb{C} \oplus \cdots \oplus \mathbb{C}}^{n \text { times }}$ and $\varphi(x)=x_{1}+$ $\cdots+x_{n}, x=\left(x_{1}, \ldots, x_{n}\right)$. Linear operators in $A$ are identified with $n \times n$ matrices in an obvious way. Let $S=\left(s_{i j}\right)$ be an $n \times n$ matrix with nonnegative real entries such that $S$ and its transpose ${ }^{t} S$ admit $1=(1, \ldots, 1)$ as an eigenvector. Then $S$ and ${ }^{t} S$ have a common eigenvalue $s>0$ for the eigenvector 1 (we exclude the trivial case $S=0$ ). Such a matrix $S$ leaves the set $A_{++} \equiv\left\{\left(x_{1}, \ldots, x_{n}\right) \in A ; x_{1}>0, \ldots, x_{n}>0\right\}$ invariant, and we can consider a function $f_{S}$ defined by

$$
f_{S}(x)=\varphi\left(S(x)^{-1} x\right), \quad x \in A_{++} .
$$

Note that $f_{S}(x)$ depends only on the ray $\mathbb{R}_{+} x=\{\alpha x ; \alpha>0\}$, and hence $f_{S}$ can be regarded as a function on the projective set $A_{++} / \mathbb{R}_{+}$.

Lemma 2. $1 \in A_{++}$is a critical point of $f_{S}$ and its Hessian is given by $2{ }^{t} S S-$ $s\left(S+{ }^{t} S\right)$.

Proof. By a direct computation.

Lemma 3. Suppose that $S$ is invertible. If the matrix $s\left(S+{ }^{t} S\right)-2{ }^{t} S S$ is positive semidefinite and its kernel is spanned by $\mathbf{1}$, then $\mathbf{1}$ is a unique (up to scalar factor) point that gives a local maximum of $f_{S}$ on $A_{++}$.

Proof. By Lemma 2, $f_{S}$ takes a local maximum at 1 . To see the uniqueness, suppose on the contrary that there is another point $a \in A_{++} \backslash \mathbb{R} 1$ of local maximum. Then Theorem 1 with $T=S^{-1}$ tells us that the Hessian at 1 should be degenerate in a direction other than 1 , which contradicts the assumption on the kernel of the Hessian. 
An $n \times n$ matrix $S$ is called cyclic if it commutes with the cyclic permutation

$$
C=\left(\begin{array}{cccc}
0 & 1 & & 0 \\
0 & \ddots & \ddots & \\
\vdots & \ddots & \ddots & 1 \\
1 & \ldots & 0 & 0
\end{array}\right)
$$

The following properties can be easily seen.

Lemma 4. (i) $S$ is cyclic if and only if $S$ is a polynomial of $C$.

(ii) Eigenvectors of a cyclic matrix $S$ are given by

$$
e_{j}=\left(\begin{array}{c}
1 \\
\zeta^{j} \\
\vdots \\
\zeta^{j(n-1)}
\end{array}\right), \quad j=0,1, \ldots, n-1,
$$

with $\zeta=e^{2 \pi i / n}$. In fact, $C e_{j}=\zeta^{j} e_{j}$.

Corollary 5. A cyclic matrix $S$ with nonnegative real entries fulfills the conditions in Lemma 3 if and only if its eigenvalues are contained in the set $\{z \in \mathbb{C}$; $|z-s / 2|<s / 2\}$ except for $s$.

Proof. Let $S=\sum_{j=0}^{n-1} s_{j} C^{j}$ with $s_{j} \geq 0$. Then the eigenvalue $s$ of $S$ for the eigenvector 1 is given by $s=\sum_{j=0}^{n-1} s_{j}$. Since eigenvalues of $S$ are of the form $\lambda=\sum_{j=0}^{n-1} s_{j} \zeta^{j}$ with $\zeta^{n}=1$, it is easy to see that $s$ is a simple root of $S$. Thus the condition in Lemma 3 is equivalent to the positivity of

$$
s(\lambda+\bar{\lambda})-2|\lambda|^{2}=2\left(\frac{s^{2}}{4}-\left|\lambda-\frac{s}{2}\right|^{2}\right)
$$

for eigenvalues $\lambda \neq s$ of $S$, proving the assertion.

Now we specialize to the case

$$
S_{n, m, s}=(s-m) 1+C^{l}+C^{l+1}+\cdots+C^{l+m-1}, \quad 1 \leq m \leq n-l, n \leq s .
$$

Lemma 6. $S_{n, m, s}$ satisfies the spectral condition in Corollary 5.

Proof. Since an eigenvalue $\lambda_{s}$ of $S_{n, m, s}$ is of the form $\lambda+(s-n)$ with $\lambda$ an eigenvalue of $S_{n, m, n}$, once the condition is proved for $s=n$, the general case follows:

$$
\begin{aligned}
\left|\lambda_{s}-s / 2\right| & =|\lambda-n / 2+(s-n) / 2| \\
& \leq|\lambda-n / 2|+(s-n) / 2<n / 2+(s-n) / 2=s / 2 .
\end{aligned}
$$

So we concentrate on the case $s=n$.

Since the spectrum of $C$ is $\left\{1, \zeta, \ldots, \zeta^{n-1}\right\}$ with $\zeta=e^{2 \pi i / n}$, eigenvalues of $S$ are given by

$$
\lambda_{j}=n-m+\zeta^{j l}+\zeta^{j(l+1)}+\cdots+\zeta^{j(l+m-1)}, \quad j=0,1, \ldots, n-1 .
$$

We need to check that these eigenvalues (except for $\lambda_{0}=n$; are contained in the open disk $|z-n / 2|<n / 2$, which can be seen as follows: For $m \leq n / 2$,

$$
\begin{aligned}
\left|\lambda_{j}-n / 2\right| & =\left|n / 2-m+\zeta^{j l}+\cdots+\zeta^{j(l+m-1)}\right| \\
& <n / 2-m+\left|\zeta^{j l}\right|+\cdots+\left|\zeta^{j(l+m-1)}\right| \\
& =n / 2-m+m=n / 2,
\end{aligned}
$$


and for $m \geq n / 2$,

$$
\begin{aligned}
\left|\lambda_{j}-n / 2\right| & =\left|n / 2-m+\zeta^{j l}+\cdots+\zeta^{j(l+m-1)}\right| \\
& =\left|n / 2-m-\zeta^{j(l+m)}-\cdots-\zeta^{j(l+n-1)}\right| \\
& <m-n / 2+\left|\zeta^{j(l+m)}\right|+\cdots+\left|\zeta^{j(l+n-1)}\right| \\
& =m-n / 2+n-m=n / 2 . \quad \square
\end{aligned}
$$

Remark. The method of the proof shows that $S_{n, m, s}$ satisfies the spectral condition in Corollary 5 whenever $s>2 \mathrm{~m}$.

Proof of the inequality. First, we give the proof of (2) for $l=1$ to get used to the reasoning. We express the summation in the left-hand side of $(2)$ by $f_{n, m, s}(x)$. Note that $f_{n, m, s}(x)=f_{S}(x)$ for $S=S_{n, m, s}$. Recall that the function $f_{n, m, s}$ is homogeneous and can be regarded as a function on the subset $A_{++} / \mathbb{R}_{+}$in the real projective space $\mathbb{P}^{n-1}$. By Lemmas 3 and $6, f_{n, m, s}(x)$ has a unique (up to scalar factor) local maximum $f_{n, m, s}(\mathbf{1})=n / s$ at $x=1$. So to prove that this is the absolute maximum, we need to examine the behaviour of $f_{n, m, s}$ at the boundary $\partial A_{++}$of $A_{++}$, where the boundary $\partial A_{++}$is taken in the projective space:

$$
\partial A_{++}=\left\{\left(x_{1}, \ldots, x_{n}\right) \in A ; x_{1} \geq 0, \ldots, x_{n} \geq 0,\left(x_{1}, \ldots, x_{n}\right) \neq(0, \ldots, 0)\right\} \text {. }
$$

According to [3], we divide the boundary into two parts: $a \in \partial A_{++}$is called in the regular boundary if the function $f_{n, m, s}(x)$ is analytically continued to a neighborhood of $a$, otherwise it is called in the singular boundary. So the problem is reduced to check (i) the behaviour of $f_{n, m, s}$ near the singular boundary and (ii) the values of $f_{n, m, s}$ at the regular boundary.

(i) Singular boundary. Let $a \in \partial A_{++}$be in the singular boundary. Then the formal expression $f_{n, m, s}(a)$ contains at least one term of the form $0 / 0$. First we consider the case where only one term of indeterminate form appears in the summand of $f_{n, m, s}(a)$. By the cyclicity, we may assume that $a_{n}=a_{1}=\cdots=$ $a_{m}=0, a_{m+1}>0, a_{n-1}>0$. When $x \in A_{++}$approaches $a$, the sum of the first $n-1$ terms in the left-hand side of (2) approaches

$$
\begin{aligned}
\frac{a_{m+1}}{(s-} & m) a_{m+1}+a_{m+2}+\cdots+a_{2 m+1} \\
& +\cdots+\frac{a_{n-m}}{(s-m) a_{n-m}+a_{n-m+1}+\cdots+a_{n-1}} \\
& +\cdots+\frac{a_{n-2}}{(s-m) a_{n-2}+a_{n-1}}+\frac{a_{n-1}}{(s-m) a_{n-1}},
\end{aligned}
$$

while the last term

$$
\frac{x_{n}}{(s-m) x_{n}+x_{1}+\cdots+x_{m}}
$$

becomes a limit of indeterminate form.

Clearly, (3) is less than

$$
\frac{a_{m+1}}{(s-m) a_{m+1}}+\cdots+\frac{a_{n-1}}{(s-m) a_{n-1}}=\frac{n-m-1}{s-m}
$$

and (4) is less than

$$
\frac{x_{n}}{(s-m) x_{n}}=\frac{1}{s-m}
$$


In total, they are less than

$$
\frac{n-m}{s-m} \leq \frac{n}{s}
$$

Thus

$$
\varlimsup_{x \rightarrow a} f_{n, m, s}(x) \leq \frac{n}{s} .
$$

We can similarly manipulate the case where $f_{n, m, s}(a)$ contains more than one term of indeterminate form $0 / 0$ and show that $(5)$ remains valid (notice the index $j$ such that $a_{j}>0$ and $a_{j-m-1}=a_{j-m}=\cdots=a_{j-1}=0$ ).

(ii) Regular boundary. Let $a \in \partial A_{++}$be in the regular boundary. We may assume that $a_{n}=0$ without loss of generality. Then

$$
\begin{aligned}
f_{n, m, s}(a)= & \frac{a_{1}}{(s-m) a_{1}+a_{2}+\cdots+a_{m+1}} \\
& +\cdots+\frac{a_{n-m-1}}{(s-m) a_{n-m-1}+a_{n-m}+\cdots+a_{n-1}} \\
& +\frac{a_{n-m}}{(s-m) a_{n-m}+a_{n-m+1}+\cdots+a_{n-1}} \\
& +\cdots+\frac{a_{n-1}}{(s-m) a_{n-1}+a_{1}+\cdots+a_{m-1}} \\
\leq & \frac{a_{1}}{(s-m) a_{1}+a_{2}+\cdots+a_{m}} \\
& +\cdots+\frac{a_{n-m-1}}{(s-m) a_{n-m-1}+a_{n-m}+\cdots+a_{n-2}} \\
& +\frac{a_{n-m}}{(s-m) a_{n-m}+a_{n-m+1}+\cdots+a_{n-1}} \\
& +\cdots+\frac{a_{n-1}}{(s-m) a_{n-1}+a_{1}+\cdots+a_{m-1}} \\
= & f_{n-1, m-1, s-1}\left(a_{1}, \ldots, a_{n-1}\right) .
\end{aligned}
$$

Thus the problem is reduced to the case $m=1$ remarking that $(n-1) /(s-1) \leq$ $n / s$. Now the estimate

$$
\begin{aligned}
f_{n, 1, s}(a) & =\frac{a_{1}}{(s-1) a_{1}+a_{2}}+\cdots+\frac{a_{n-2}}{(s-1) a_{n-2}+a_{n-1}}+\frac{a_{n-1}}{(s-1) a_{n-1}} \\
& <\frac{a_{1}}{(s-1) a_{1}}+\cdots+\frac{a_{n-1}}{(s-1) a_{n-1}}=\frac{n-1}{s-1} \leq \frac{n}{s}
\end{aligned}
$$

completes the analysis at the regular boundary, proving the inequality (2) for $l=1$.

Remark. From the discussions at the singular boundary, we know that the inequality (2) with $l=1$ breaks down for $s<n$. In fact, maximizing (3) and (4), respectively, we can find a sequence $x \in A_{++}$such that

$$
\varlimsup_{x \rightarrow a} f_{n, m, s}(x)=\frac{n-m}{s-m}>\frac{n}{s} \text {. }
$$

This fact, combined with the remark after Lemma 6 , shows that the spectral condition on the Hessian at 1 does not control the behaviour of the function $f_{n, m, s}$ at the boundary. 
Generalization. Now the general case will be dealt with. Let us begin with the case $m=1$. If $n$ and $l$ are relatively prime, then by changing the variables $x_{j}$ 's according to the permutation

$$
\left(\begin{array}{ccccc}
1 & 2 & 3 & \ldots & n \\
l & 2 l & 3 l & \ldots & n l
\end{array}\right)
$$

(the second line is calculated modulo $n$ ) the problem is reduced to the case $l=1$. If $n$ and $l$ have the greatest common divisor $d \geq 2$, then the summand in $f_{n, m, s}(a)$ splits into $d$-parts and the problem is reduced to the case $d=1$, just discussed.

So from now on, we assume that $m \geq 2$. Then most of the above argument is valid without changes; the only exception is in the discussion at the singular boundary, where we need to show that at least $m$ terms become 0 in the summation of $f_{n, m, s}(a)$. Since $f_{n, m, s}(a)$ contains at least one term of indeterminate form $0 / 0$, we may assume that $a_{1}=\cdots=a_{m}=0$. We complete the analysis at the singular boundary by showing that the number of $1 \leq j \leq n$ satisfying

$$
a_{j}=0, \quad\left(a_{j+l}, a_{j+l+1}, \ldots, a_{j+l+m-1}\right) \neq(0,0, \ldots, 0),
$$

is $\geq m$. To see this, it is convenient to introduce the following terminology. A successive subsequence $a_{p}, a_{p+1}, \ldots, a_{q}$ of $\left\{a_{j}\right\}_{j \geq 1}$ is called a null-string if $a_{p}=a_{p+1}=\cdots=a_{q}=0$ and $a_{p-1}>0, a_{q+1}>0$.

Consider a null-string of maximum length. By the cyclic nature, we may assume that it is of the form $\left\{a_{1}, \ldots, a_{k+m}\right\} \quad(k \geq 0)$. If

$$
\left(a_{j+l}, a_{j+l+1}, \ldots, a_{j+l+m-1}\right) \neq(0,0, \ldots, 0), \quad k+1 \leq \forall j \leq k+m,
$$

then the claim clearly holds. So we restrict ourselves to the case

$$
\left(a_{j+l}, a_{j+l+1}, \ldots, a_{j+l+m-1}\right)=(0,0, \ldots, 0),
$$

for some $j$ in $\{k+1, k+2, \ldots, k+m\}$.

Let $j_{2}$ be the maximum of such a $j$ and $j_{1} \leq j_{2}$ be such that

$$
a_{j_{1}+l}, \ldots, a_{j_{2}+l}, \ldots, a_{j_{2}+l+m-1}=(0, \ldots, 0)
$$

and $a_{j_{1}+l-1} \neq 0$. Note that $j_{1} \geq j_{2}-k$ as the maximum length of null-strings is $k+m$. Since $(* *)$ is a null string for $j_{2}<k+m$, we see that the index $j$ in the range

$$
\max \left(1, j_{1}-m\right) \leq j \leq j_{1}-1 \text { or } j_{2}+1 \leq j \leq k+m,
$$

satisfies the condition $(*)$ and the number of $j$ in this range is given by

$$
\begin{aligned}
& \min \left(j_{1}-1+k+m-j_{2},\left(j_{1}-1\right)-\left(j_{1}-m-1\right)+k+m-j_{2}\right) \\
& \quad=\min \left(j_{1}+k-j_{2}+m-1, m+k+m-j_{2}\right) \\
& \quad \geq \min (m-1, m)=m-1
\end{aligned}
$$

because $j_{1} \geq j_{2}-k$ and $j_{2} \leq k+m$.

Thus, if there exist two null-strings of length $k+m$, then the number $N$ of $j$ satisfying $(*)$ is estimated as

$$
N \geq 2(m-1) \geq m
$$

(note that $m \geq 2$ ). 
Now suppose that $N=m-1$ and there is the unique null-string of maximum length. Since $N=m-1$, the above reasoning shows that $j_{1}-m \leq 0$ and $j_{1}+k-j_{2}=0$, which implies that the length of the null string containing $(* *)$ is $\geq m+k$. Since the string of length $m+k$ is maximal and unique, we should have $j_{1}+l=1(\bmod n)$, i.e., $j_{1}=n+1-l$. Then $n+1-l \leq m$, which contradicts the assumption $m \leq n-l$. Thus we have completed the proof of (2).

Conjecture. Let $n$ be a positive integer, $s$ be a positive real number, and $I$ be a subset of $\{1, \ldots, n-1\}$. As a generalization of the inequality $(2)$, it is natural to expect the inequality

$$
\sum_{j=1}^{n} \frac{x_{j}}{(s-|I|) x_{j}+\sum_{i \in I} x_{i+j}} \leq \frac{n}{s},
$$

where $|I|$ denotes the number of elements in $I$.

By a calculation based on the arguments in the proof of (2), we can check the validity of the above inequality in the most simple case $n=4, I=\{1,3\}$, which is not included in (2). The author has, however, no other clues on this problem.

\section{ACKNOWLEDGMENT}

The author is indebted to K. Tanahashi for calling attention to Nowasad's work [3] and much useful information on the present subject and to F. Hiai for pointing out some defects in the original description of the proof of (2).

\section{REFERENCES}

1. T. Ando, Positivity of certain maps, Seminar Notes, 1985.

2. M. D. Choi, Positive linear maps on $C^{*}$-algebras, Canad. J. Math. 24 (1972), 520-529.

3. P. Nowosad, Isoperimetric eigenvalue problems in algebras, Comm. Pure Appl. Math. 21 (1968), 401-465.

4. H. Osaka, Indecomposable positive maps in low dimensional matrix algebras, Linear Algebra Appl. 153 (1991), 73-83.

5. _ A series of absolutely indecomposable positive maps in matrix algebras, preprint.

6. $\mathrm{K}$. Tanahashi and J. Tomiyama, Indecomposable positive maps in matrix algebras, Canad. Math. Bull. 31 (1988), 308-317.

Department of Mathematics, College of General Education, Tohoku University, SENDAI 980, JAPAN

E-mail address: e22236@cctu.cc.tohoku.ac.jp 